\title{
Effect of Quintessential and Magnetic Field on GUP Modified Hawking Radiation
}

\author{
Rimsha Babar, ${ }^{1, *}$ Wajiha Javed, ${ }^{1,+}$ and Ali Övgün ${ }^{2,3, \ddagger}$ \\ ${ }^{1}$ Department of Mathematics, University of Education, \\ Township, Lahore-54590, Pakistan. \\ ${ }^{2}$ Instituto de Física, Pontificia U niversidad C atólica de Valparaíso, C asilla 4950, V alparaíso, Chile \\ ${ }^{3}$ Physics Department, Faculty of Arts and Sciences, Eastern Mediterranean University, Famagusta, North Cyprus, via Mersin 10, Turkey
} (Dated: August 6, 2019)

In this paper, we investigate the Hawking radiation process by using the quantum tunneling phenomenon of massive spin- 1 (W-bosons) and spin- 0 particles from $(2+1)$ dimensional Black Hoke with quintessential and magnetic field. For this purpose, using Hamilton-Jacobi ansatz, we apply the WKB approximation to the field e quations of $\mathrm{m}$ assive $\mathrm{c}$ harged $\mathrm{v}$ ector $\mathrm{p}$ a rticles. We $\mathrm{g}$ et $\mathrm{t}$ he $\mathrm{r}$ equired tunneling rate of radiated particles and obtain their corresponding Hawking temperature $T_{h}$. In order to study the quantum gravity effects, we utilize the generalized Proca and Klein-Gordan equations incorporating the generalized uncertainty principle (GUP) and recover the accompanying quantum corrected temperature $T_{h}^{\prime}$.

PACS numbers: 04.40.-b, 95.30.Sf, 98.62.Sb

Keywords: Hawking radiation; quantum tunneling; quantum corrections; Proca equation; modified KleinGordan equation

\section{INTRODUCTION}

The study of quantum mechanical effects within the foundation of classical general relativity provide numerous interesting phenomenon, which play very important part to understand the quantum gravity theories. In quantum theory, the black holes (BHs) evaporation as a result of Hawking radiation [1] is one of the important phenomenon. During the study of radiation process the researchers try to combine the gravitation within $\mathrm{BH}$ thermodynamics and the quantum mechanics $[2,3]$. In order to study the Hawking radiation phenomenon, many techniques have been proposed in literature. Many researchers have studied these radiations for well known BHs [4]-[42]. These radiations can be investigated by the semiclassical approach, depend upon quantum tunneling method of a particle through outer horizon of $\mathrm{BH}$ from inside to outside $[43,44]$. The tunneling approach based on two different techniques, null geodesic [45]-[47] and Hamilton Jacobi method [48]. These techniques derive the imaginary part of the classical action $(I)$ from outer horizon by following the classically forbidden trajectory of a particle. The tunneling probability from a $\mathrm{BH}$ in both techniques can be defined as

$$
\Gamma_{\mathcal{P}}=e^{-\frac{2}{\hbar} \operatorname{ImI}} .
$$

This probability formula provides the Hawking temperature of the $\mathrm{BH}$. The presence of a minimal observable length is a common factor in different quantum gravity theories e.g., loop quantum gravity, noncommutative geometry and string theory [49]-[52]. The generalized uncertainty principle (GUP) is a straightforward way for understanding this minimal length [53].The generalized commutation relation can be given as $\left[x_{u}, \mathfrak{p}_{v}\right]=i \hbar \delta_{u v}\left[1+\beta \mathfrak{p}^{2}\right]$, where $x_{u}$ is the modified position operator while $\mathfrak{p}_{v}$ stands for modified momentum operator.

The GUP relation can be expressed in the following form

$$
\Delta x \Delta \mathfrak{p} \geq \frac{\hbar}{2}\left[1+\beta\left(\Delta \mathfrak{p}^{2}\right)\right]
$$

where $\beta=\beta_{\circ} / \tilde{M}_{\mathfrak{p}^{2}}$. $\beta_{\circ}$ and $\tilde{M}_{\mathfrak{p}}$ are the dimensionless parameter and Plank mass, respectively. The GUP relations play a vital role in understanding the nature of $\mathrm{BHs}$, while the quantum effects may be considered vital effects around

\footnotetext{
*Electronic address: rimsha.babar10@gmail.com

†Electronic address: wajiha.javed@ue.edu.pk; wajihajaved84@yahoo.com

‡Electronic address: ali.ovgun@pucv.cl; https : / /www. aovgun.com
} 
the event horizon of a BH. By utilizing the quantum tunneling method, the quantum effects incorporating GUP relations with thermodynamical properties for different spins of BHs have been widely studied [54]-[84]. Later cosmic observations emphatically suggest that our universe is right now experiencing a stage of accelerated expansion [85], likely determined by some exotic form known dark energy. In spite of the mounting observational proof, the nature and cause of dark energy is still like a puzzle and it has become a matter of vivid discussion. Quintessence is one of the logical possibility for dark energy with negative pressure [86]. It is evolving, dynamic and spatially heterogeneous element. By comparison within the present context, it would be the fifth dynamical element that has affected the expansion of the Universe, additionally to the already known photons, baryons, dark matter and leptons. It can be described by its equation of state i.e., $\left(\omega_{q}=p / \rho\right)$, where $\rho$ and $p$ denotes the energy density and pressure for quintessential field, respectively. Moreover, most of the models have the range $0 \geq \omega_{q}>-1$, though a cosmological consistent has $\omega_{q}=-1$. The accelerating expansion depend upon the value of $\omega_{q}$, as smaller its value gives the more accelerating effect. The Einstein field equations for four and $d$-dimensional BHs with quintessential field have been studied $[87,88]$.

The investigation of $(2+1)$-dimensional BHs gives an important knowledge in comprehension of lowdimensional gravity theories and their quantum partners. The Banados-Teitelboim-Zanelli (BTZ) BH is such a model in $(2+1)$-dimensional gravity [89]. This BH is surprisingly comparative to $(3+1)$-dimensional BH. Just like the Kerr $\mathrm{BH}$, it has an outer and inner horizon. It may be completely portrayed by charge, mass and angular momentum. Additionally, it has thermodynamical properties closely resembling to the $(3+1)$-dimensional BH. The BTZ solution has been additionally discussed in the domain of $(2+1)$-dimensional quantum gravity. Moreover, it will be very interesting to study the BTZ-like BH with magnetic field. It is a well known fact that electric field is related to the temporal component of gauge potential $A_{t}$, whereas the electric field is linked with the angular part of the gauge potential $A_{\varphi}$. These types of BHs, only magnetic gauge can be used to derive BH solutions. It is accepted that the BHs in $(2+1)$-dimensions will generally provide a basic laboratory and an outstanding comprehension for examining the general perspectives of $\mathrm{BH}$ physics. The other main reason and motivation to choose $(2+1)$-dimensional $\mathrm{BHs}$ is the presence of AdS/CFT correspondence [90], that relates the thermodynamical properties of BHs with AdS/CFT duality. In recent years, the researcher has paid a much attention to analyze the thermal properties of $(2+1)$-dimensional $\mathrm{BHs}$, particularly with a space-time having non-vanishing cosmological constant. The main purpose of this article is to investigate the Hawking radiation phenomenon with GUP effects under quintessential and magnetic field from $(2+1)$-dimensional BHs. For this purpose, firstly, we consider a $(2+1)$-dimensional BH with quintessential field. Then by utilizing the Hamilton Jacobi technique, we calculated the tunneling probability and Hawking temperature for spin-1 particles. Moreover, we have analyzed the corrected Hawking temperture for spin-1 and spin-0 particles. Further, we consider a magnetically charged version of BTZ-like BH. We have studied the modified tunneling probability and corrected Hawking temperature for spin-1 and spin-0 particles for BTZ-like BH with magnetic field. The paper is outlined as: Sec. II contains the information about metric of $(2+1)$-dimensional BH with quintessential field. Sec. III is devoted for study of tunneling probability and Hawking temperature for quintessential BH for vector particles. In Sec. IV, we investigate the modified tunneling probability and corresponding temperature for vector particles from quintessential BH under GUP effects. $\mathbf{V}$ is based on the quantum gravity effects for vector particles from BTZ-like magnetic BH. In Sec. VI, we investigate the quantum corrections for scalar particles from quintessential BH. Sec. VII represents the modified tunneling rate and related effective Hawking temperature for spin-0 particles from BTZ-like BH with magnetic field. In Sec. VIII, we summarize the final results of our work.

\section{METRIC FOR (2+1) DIMENSIONAL BLACK HOLE WITH QUINTESSENCE}

The line element for three dimensional BH surrounded by quintessence is defined as [54]

$$
d s^{2}=-\frac{r^{2}}{l^{2}} g(r) d t^{2}+\frac{l^{2}}{r^{2}} g(r)^{-1} d r^{2}+r^{2} d x^{2}
$$

where

$$
g(r)=1-\left(\frac{r_{+}}{r}\right)^{\rho}, \quad \rho=2\left(1+\omega_{q}\right)
$$

and $r_{+}=\left(M l^{2}\right)^{1 / \rho}$ represents the event horizon, $M$ specifies the mass of $\mathrm{BH}, \omega_{q}$ shows the quintessence parameter with range $-1<\omega_{q}<-\frac{1}{3}$, $l$ stands for AdS radius which belongs to cosmological constant by $l^{2}=-1 / \Lambda$, moreover $r$ and $x$ are the radial and planar coordinates, respectively, lies between $(0, \infty)$.

The line element (3) can also be rewritten as

$$
d s^{2}=-X(r) d t^{2}+\frac{1}{Y(r)} d r^{2}+Z(r) d x^{2}
$$


the above metric functions can be defined as follows

$$
X(r)=\frac{r^{2}}{l^{2}}\left[1-\left(\frac{r_{+}}{r}\right)^{\rho}\right], Y(r)=\frac{l^{2}}{r^{2}}\left[1-\left(\frac{r_{+}}{r}\right)^{\rho}\right]^{-1}, Z(r)=r^{2} .
$$

It is important to mention here that for $\omega_{q}=0$ the line element reduces into BTZ BH metric [55].

\section{VECTOR PARTICLES TUNNELING}

This section is devoted to study the tunneling spectrum for spin-1 particles at event horizon of BH, by using Proca equation under vector field $\Psi_{v}$. The Proca equation can be expressed as [? ]

$$
\frac{1}{\sqrt{-\tilde{g}}} \partial_{u}\left(\sqrt{-\tilde{g}} \Psi^{v u}\right)+\frac{m^{2}}{\hbar^{2}} \Psi^{v}=0, \quad(u, v=0,1,2),
$$

here $\tilde{g}$ denotes the determinant of the metric and $m$ is mass of emitted particle. Also, $\Psi^{u v}$ represents anti-symmetric tensor which can be defined as

$$
\Psi_{v u}=\mathcal{D}_{v} \Psi_{u}-\mathcal{D}_{u} \Psi_{v}=\partial_{v} \Psi_{u}-\partial_{u} \Psi_{v}
$$

Here, $\mathcal{D}_{u}$ stands for covariant derivative. We will get the similar results for $W^{+}$and $W^{-}$bosons, the equation of motion of tunneling process for both particles is same. But, here we only deal with $W^{+}$boson particles.

The wave function for spin-1 particles by considering the WKB approximation is defined as

$$
\Psi_{v}=\left(a_{0}, a_{1}, a_{2}\right) \exp \left[\frac{i}{\hbar} I(t, r)\right]
$$

where the action $I(t, r)$ is given as

$$
I(t, r)=I_{0}(t, r)+\hbar I_{1}(t, r)+\hbar^{2} I_{2}(t, r)+\ldots,
$$

After substituting the covariant components $\psi_{0}=-X \psi^{0}, \psi_{1}=(1 / Y) \psi^{1}, \psi_{2}=Z \psi^{2}$, the metric (4) and Eq. (7) into Eq.(5) neglecting the higher terms of $\hbar$, we obtain the following set of equations

$$
\begin{aligned}
& Y\left[a_{0}\left(\partial_{r} I\right)^{2}-a_{1}\left(\partial_{t} I\right)\left(\partial_{r} I\right)\right]+\frac{1}{Z}\left[a_{0}\left(\partial_{x} I\right)^{2}-a_{2}\left(\partial_{t} I\right)\left(\partial_{x} I\right)\right]+m^{2} a_{0}=0 \\
& \frac{1}{X}\left[a_{0}\left(\partial_{t} I\right)\left(\partial_{r} I\right)-a_{1}\left(\partial_{r} I\right)^{2}\right]+\frac{1}{Z}\left[a_{1}\left(\partial_{x} I\right)^{2}-a_{2}\left(\partial_{r} I\right)\left(\partial_{x} I\right)\right]+m^{2} a_{1}=0 \\
& \frac{1}{X}\left[a_{0}\left(\partial_{t} I\right)\left(\partial_{x} I\right)-a_{2}\left(\partial_{t} I\right)^{2}\right]+Y\left[a_{2}\left(\partial_{r} I\right)^{2}-a_{1}\left(\partial_{x} I\right)\left(\partial_{r} I\right)\right]+m^{2} a_{2}=0
\end{aligned}
$$

We consider a particle's action in the following form

$$
I=-E t+R(r)+j x+C,
$$

where $E$ denotes the energy of emitted particles and $C$ represents the complex constant. The above set of equations gives a $3 \times 3$ matrix equation, which can be given as

$$
\aleph\left(a_{0}, a_{1}, a_{2}\right)^{T}=0,
$$

where

$$
\aleph=\left(\begin{array}{ccc}
Y\left(\partial_{r} R\right)^{2}+\frac{\left(\partial_{x} j\right)^{2}}{Z}+m^{2} & Y E \partial_{r} R & \frac{E \partial_{x} j}{Z} \\
\frac{E \partial_{r} R}{X} & \frac{E^{2}}{X}-\frac{\left(\partial_{x} j\right)^{2}}{Z}-m^{2} & \frac{\left(\partial_{r} R\right) \partial_{x} j}{Z} \\
\frac{E \partial_{x} j}{X} & Y\left(\partial_{r} R\right) \partial_{x} j & \frac{E^{2}}{X}-B\left(\partial_{r} R\right)^{2}-m^{2}
\end{array}\right)
$$

In order to obtain the non-trivial solution, we set $\operatorname{det} \aleph=0$ and obtain

$$
R_{ \pm}(r)= \pm \sqrt{\frac{E^{2}-X(r)\left(m^{2}+\frac{\left(\partial_{x} j\right)^{2}}{Z(r)}\right)}{X(r) Y(r)}} d r
$$


The $R_{+}$and $R_{-}$represents the outgoing motion (with momentum $\partial_{r} I>0$ ) and ingoing motion (with momentum $\left.\partial_{r} I<0\right)$, respectively.

The $R_{ \pm}$possesses a simple pole at $r=r_{+}$and it gives the same imaginary part for both outgoing/ingoing solutions.

$$
\begin{aligned}
R_{ \pm}(r) & = \pm \frac{i \pi E}{X^{\prime}\left(r_{+}\right)}, \\
I m R_{ \pm}(r) & = \pm \frac{\pi E}{X^{\prime}\left(r_{+}\right)} .
\end{aligned}
$$

The evaporation process in BHs is a quantum process [1] and the absorption/emission probabilities for crossing the event horizon in and out can be defined as [57]

$$
\begin{aligned}
\mathcal{P}[\text { emission }] & =\mathcal{P}_{+}=-\exp \left[-\frac{2}{\hbar}\left(\operatorname{Im} R_{+}+\operatorname{Im} C\right)\right], \\
\mathcal{P}[\text { absorption }] & =\mathcal{P}_{-}=\exp \left[-\frac{2}{\hbar}\left(\operatorname{Im} R_{+}+\operatorname{Im} C\right)\right] .
\end{aligned}
$$

It is important to mention here that, a factor of two problem arises and to solve this problem we choose the ingoing probability as $\left(\mathcal{P}_{-} \simeq e^{-\frac{2}{\hbar} \operatorname{Im} R_{-}}=1\right)$. Due to the fact that $R_{+}=-R_{-}$, we have $\operatorname{ImC}=\operatorname{Im} R_{+}$and therefore from Eq. (18), we get

$$
\mathcal{P}[\text { emission }]=\exp \left[-\frac{4}{\hbar}\left(\operatorname{Im} R_{+}\right)\right],
$$

So, the total tunneling probability for radiated particles from inside to outside can be defined as follows

$$
\begin{aligned}
& \Gamma_{\mathcal{P}}=\frac{\mathcal{P}[\text { emission }]}{\mathcal{P}[\text { absorption }]} \simeq \exp \left[-\frac{4}{\hbar}\left(\operatorname{Im} R_{+}\right)\right] \\
& \Gamma_{\mathcal{P}}=\exp \left[-\frac{4 \pi E}{\hbar X^{\prime}\left(r_{+}\right)}\right]=\exp \left[-\frac{8 \pi l^{2} E}{\hbar \rho^{2} r_{+}}\right] .
\end{aligned}
$$

The surface gravity $\kappa$ for the given $\mathrm{BH}$ can be calculated as

$$
\kappa=\left[\frac{\rho^{2} r_{+}}{4 \pi l^{2}}\right] .
$$

In order to obtain the corresponding Hawking temperature, we use Boltzmann factor $\Gamma_{\mathcal{P}}=\exp \left[E / T_{h}\right]$ and get

$$
T_{h}=\frac{\kappa}{2 \pi}=\left[\frac{\rho^{2} r_{+}}{8 \pi l^{2}}\right], \quad(\hbar=1) .
$$

This is the required Hawking temperature. It depends upon the curvature radius $l$ and quintessence parameter $\omega_{q}$ (i.e., $\rho=2\left(1+\omega_{q}\right)$. For the different values of $\omega_{q}$, we can derive the Hawking temperature in the following form:

when $\omega_{q}=-1 / 3$

$$
T_{h}=\left[\frac{2 r_{+}}{9 \pi l^{2}}\right]
$$

when $\omega_{q}=-1 / 2$

$$
T_{h}=\left[\frac{r_{+}}{18 \pi l^{2}}\right]
$$

when $\omega_{q}=-2 / 3$

$$
T_{h}=\left[\frac{r_{+}}{8 \pi l^{2}}\right] .
$$




\section{QUANTUM GRAVITY EFFECTS FOR SPIN-1 PARTICLES}

In this section, we study the corrected tunneling rate for spin-1 particles through the horizon, we choose a Lagrangian equation under quantum gravity effects. The GUP modified Lagrangian equation for spin-1 particles with vector field $\Psi_{u}$ can be given as [58]

$$
£^{G U P}=-\frac{1}{2}\left[\mathfrak{D}_{u} \Psi_{v}-\mathfrak{D}_{v} \Psi_{u}\right]\left[\mathfrak{D}^{u} \Psi^{v}-\mathfrak{D}^{v} \Psi^{u}\right]-\frac{m^{2}}{\hbar^{2}} \Psi^{v} \Psi_{u}
$$

The modified equation of motion for bosons particles can be defined as follows

$$
\partial_{u}\left(\sqrt{-\tilde{g}} \Psi^{u v}\right)+\hbar^{2} \beta \partial_{0} \partial_{0} \partial_{0}\left(\sqrt{-\tilde{g}} g^{00} \Psi^{0 v}\right)-\hbar^{2} \beta \partial_{i} \partial_{i} \partial_{i}\left(\sqrt{-\tilde{g}} g^{i i} \Psi^{i v}\right)-\sqrt{-\tilde{g}} \frac{m^{2}}{\hbar^{2}} \Psi^{v}=0
$$

Here, $m$ shows the mass of emitted particle. The generalized anti-symmetric tensor $\Psi^{u v}$ can be defined as

$$
\Psi^{u v}=\left(1-\beta \hbar^{2} \partial_{u}^{2}\right) \partial_{u} \Psi_{v}-\left(1-\beta \hbar^{2} \partial_{v}^{2}\right) \partial_{v} \Psi_{u}
$$

The modified tensor $\Psi^{i u}, i=1,2$ stands for spatial components and $\Psi^{0 u}, 0$ states the time coordinate.Also, the correction parameter $\beta$ can be given as $\beta=\frac{3}{M_{f}^{2}}$, where $M_{f}$ denotes the minimal length.

The wave function for emitted particles with vector field $\Psi^{u}$ can be expressed as

$$
\Psi_{v}=\left(a_{0}, a_{1}, a_{2}\right) \exp \left[\frac{i}{\hbar} I(t, r)\right]
$$

By using the metric components from Eq. (4) and values from Eqn.(30) and (31) into Eq. (29), we get a set of equations

$$
\begin{aligned}
& Y\left[a_{0}\left(\partial_{r} I\right)^{2} \tilde{P}_{1}^{2}-a_{1}\left(\partial_{r} I\right)\left(\partial_{t} I\right) \tilde{P}_{1} \tilde{P}_{0}\right]+\frac{1}{Z}\left[a_{0}\left(\partial_{x} I\right)^{2} \tilde{P}_{2}^{2}-a_{2}\left(\partial_{x} I\right)\left(\partial_{t} I\right) \tilde{P}_{2} \tilde{P}_{0}\right]+a_{0} m^{2}=0 \\
& -\frac{1}{X}\left[a_{1}\left(\partial_{t} I\right)^{2} \bar{P}_{0}^{2}-a_{0}\left(\partial_{t} I\right)\left(\partial_{r} I\right) \tilde{P}_{0} \tilde{P}_{1}\right]+\frac{1}{Z}\left[a_{1}\left(\partial_{x} I\right)^{2} \tilde{P}_{2}^{2}-a_{2}\left(\partial_{x} I\right)\left(\partial_{r} I\right) \tilde{P}_{2} \tilde{P}_{1}\right]+a_{1} m^{2}=0 \\
& -\frac{1}{X}\left[a_{2}\left(\partial_{t} I\right)^{2} \tilde{P}_{0}^{2}-a_{0}\left(\partial_{t} I\right)\left(\partial_{x} I\right) \tilde{P}_{0} \tilde{P}_{2}\right]+Y\left[a_{2}\left(\partial_{r} I\right)^{2} \tilde{P}_{1}^{2}-a_{1}\left(\partial_{r} I\right)\left(\partial_{x} I\right) \tilde{P}_{1} \tilde{P}_{2}\right]+a_{2} m^{2}=0
\end{aligned}
$$

where

$$
\begin{aligned}
& \tilde{P}_{0}=1+\beta \frac{1}{X(r)}\left(\partial_{t} I\right)^{2}, \quad \tilde{P}_{1}=1+\beta Y(r)\left(\partial_{r} I\right)^{2} \\
& \tilde{P}_{2}=1+\beta \frac{1}{Z(r)}\left(\partial_{x} I\right)^{2} .
\end{aligned}
$$

By assuming the separation of variables technique

$$
I=-E t+R(r)+j x+C
$$

where $E$ denotes the energy of emitted particles and $C$ represents the complex constant. After inserting Eq.(36) in the above set of equations, a matrix equation can given as

$$
\aleph\left(a_{0}, a_{1}, a_{2}\right)^{T}=0,
$$

where

$$
\aleph=\left(\begin{array}{ccc}
Y R^{\prime 2} \tilde{P}_{1}^{2}+\frac{j^{2}}{Z} \tilde{P}_{2}^{2}+m^{2} & Y E R^{\prime} \tilde{P}_{0} \tilde{P}_{1} & \frac{E j \tilde{P}_{0} \tilde{P}_{2}}{Z} \\
\frac{-E R^{\prime} \tilde{P}_{0} \tilde{P}_{1}}{X} & \frac{-E^{2} \tilde{P}_{0}^{2}}{X}+\frac{j^{2} \tilde{P}_{2}^{2}}{Z}+m^{2} & \frac{-j R^{\prime} \tilde{P}_{1} \tilde{P}_{2}}{Z} \\
\frac{-E j \tilde{P}_{0} \tilde{P}_{2}}{X} & -Y j R^{\prime} \tilde{P}_{1} \tilde{P}_{2} & \frac{-E^{2} \tilde{P}_{0}^{2}}{X}+Y R^{\prime 2} \tilde{P}_{1}^{2}+m^{2}
\end{array}\right)
$$

where $R^{\prime}=\partial_{r} R$. When we set $\operatorname{det} \aleph=0$, from Eq.(38) then we obtain a non-trivial solution in the form

$$
\partial_{r} R_{ \pm}= \pm \sqrt{\frac{-m^{2}}{X(r)}+\frac{E^{2}}{X(r)^{2}}-\frac{j^{2}}{X(r) Z(r)}}\left(1+\frac{\mathfrak{F}_{1}}{\mathfrak{F}_{2}} \beta\right)
$$


where

$$
\begin{aligned}
\mathfrak{F}_{\perp} & =-3 m^{4} X(r) Z(r)+6 E^{2} m^{2} Z(r)-6 m^{2} j^{2} X(r)-\frac{6 j^{4} X(r)}{Z(r)}+6 E^{2} j^{2} \\
& -\frac{7 X(r) j^{2}}{Z(r)}-\frac{3 X(r) j^{2}}{2 m^{2} Z(r)^{2}}+\frac{3 X(r) j^{2}}{2 m^{2} Z(r)^{2}}, \\
\mathfrak{F}_{2} & =-m^{2} X(r) Z(r)+E^{2} Z(r)-j^{2} X(r) .
\end{aligned}
$$

After integrating the Eq.(39), we calculate the imaginary part of the radial action in the following form

$$
\operatorname{Im} R_{ \pm}= \pm \iota \pi \frac{E}{X^{\prime}(r)}(1+\beta \chi)
$$

where $\chi>0$ and can be given as

$$
\chi=6 m^{2}+\frac{6 j^{2}}{r_{+}^{2}} .
$$

Here, $R_{+} / R_{-}$stands for radial functions for the outgoing/incoming particles, respectively. The tunneling rate for spin-1 particles at event horizon can be derived as

$$
\begin{aligned}
& \Gamma_{\mathcal{P}}=\frac{\mathcal{P}[\text { emission }]}{\mathcal{P}[\text { absorption }]} \simeq \exp \left[-\frac{4}{\hbar}\left(\operatorname{ImR_{+}}\right)\right] \\
& \Gamma_{\mathcal{P}}=\exp \left[-\frac{4 \pi E}{\hbar X^{\prime}\left(r_{+}\right)}(1+\beta \chi)\right]=\exp \left[-\frac{8 \pi l^{2} E}{\hbar \rho^{2} r_{+}}(1+\beta \chi)\right] .
\end{aligned}
$$

The corresponding effective Hawking temperature can be calculated as follows

$$
T_{h}^{\prime}=\frac{\rho^{2} r_{+}}{8 \pi l^{2}(1+\beta \chi)}=T_{h}(1-\beta \chi),
$$

where

$$
T_{h}=\frac{\rho^{2} r_{+}}{8 \pi l^{2}}
$$

this is the original Hawking temperature of the given $\mathrm{BH}$. Equation (43) represents the effective Hawking temperature under quantum gravity effects.

In case of quantum corrections we can observe the stopping of $\mathrm{BH}$ evaporation. Quantum corrections decelerates the increase in Hawking temperature during the radiation phenomenon. During the evaporation process, we can achieve a balance state, where the evaporation stops and remnants are left. When $(\beta=0)$, we can get the original Hawking temperature $T_{h}$ for given $\mathrm{BH}$ surrounded by the quintessence. For different values of quintessence, we can obtain the corrected Hawking temperature as follows:

when $\omega_{q}=-1 / 3$

$$
T_{h}^{\prime}=\left[\frac{2 r_{+}}{9 \pi l^{2}}(1-\beta \chi)\right]
$$

when $\omega_{q}=-1 / 2$

$$
T_{h}^{\prime}=\left[\frac{r_{+}}{18 \pi l^{2}}(1-\beta \chi)\right]
$$

when $\omega_{q}=-2 / 3$

$$
T_{h}^{\prime}=\left[\frac{r_{+}}{8 \pi l^{2}}(1-\beta \chi)\right] .
$$




\section{QUANTUM GRAVITY EFFECTS FOR CHARGED BTZ-LIKE MAGNETIC BLACK HOLE}

The line element for $(2+1)$ dimensional BTZ-like BH with magnetic charge $q_{m}$ can be defined as [9]

$$
d s^{2}=-\frac{r^{2}}{l^{2}} d t^{2}+\frac{1}{G(r)} d r^{2}+l^{2} G(r) \tau^{2} d \varphi^{2}
$$

where $G(r)$ is the metric function, $l$ denotes the radius curvature with negative cosmological constant $\Lambda=-\frac{1}{l^{2}}, r$ represents the radial coordinate and

$$
G(r)=\frac{r^{2}}{l^{2}}-\left[\tilde{M}+8 q_{m}^{2} l^{2} \tau^{2} \ln \left(\frac{r}{l}\right)\right]
$$

where $\tilde{M}$ shows the mass of the $\mathrm{BH}$ and $\tau$ is the arbitrary parameter. The Eq.(47) can be rewritten in the following form

$$
d s^{2}=\tilde{X}(r) d t^{2}+\tilde{Y}(r) d r^{2}+\tilde{Z}(r) d \varphi^{2}
$$

where the metric functions $\tilde{X}(r), \tilde{Y}(r)$ and $\tilde{Z}(r)$ can be given as

$$
\tilde{X}(r)=-\frac{r^{2}}{l^{2}}, \tilde{Y}(r)=\frac{1}{G(r)}, \quad \tilde{Z}(r)=l^{2} G(r) \tau^{2} .
$$

The gauge potential of $\mathrm{BH}$ is given as follows

$$
A_{\mu}=-2 q_{m} l^{2} \tau^{2} k(r) \delta_{\mu}^{\varphi}
$$

here $A_{\varphi}$ is the only non-zero component due to magnetic field and $k(r)$ represents the arbitrary function of $r$.

In order to investigate the corrected tunneling rate and Hawking temperature for BTZ-like charged magnetic BH, we consider the following modified wave equation for massive bosons as

$$
\begin{aligned}
& \partial_{u}\left(\sqrt{-\tilde{g}} \Psi^{v u}\right)+\sqrt{-\tilde{g}} \frac{m^{2}}{\hbar^{2}} \Psi^{v}+\sqrt{-\tilde{g}} \frac{l}{\hbar} e A_{u} \Psi^{v u}+\sqrt{-\tilde{g}} \frac{l}{\hbar} e \tilde{F}^{v u} \Psi_{u}+\hbar^{2} \beta \partial_{0} \partial_{0} \partial_{0} \\
& \left(\sqrt{-\tilde{g}} g^{00} \Psi^{0 v}\right)-\hbar^{2} \beta \partial_{i} \partial_{i} \partial_{i}\left(\sqrt{-\tilde{g}} g^{i i} \Psi^{i v}\right)=0,
\end{aligned}
$$

The anti-symmetric tensor for charged particles can be defined as

$$
\begin{aligned}
& \Psi_{v u}=\left(1-\hbar^{2} \beta \partial_{v}^{2}\right) \partial_{v} \Psi_{u}-\left(1-\hbar^{2} \beta \partial_{u}^{2}\right) \partial_{u} \Psi_{v}+\left(1-\hbar^{2} \beta \partial_{v}^{2}\right) \frac{\iota}{\hbar} e A_{v} \Psi_{u} \\
&-\left(1-\hbar^{2} \beta \partial_{u}^{2}\right) \frac{\iota}{\hbar} e A_{u} \Psi_{v}, \text { and } \\
& \tilde{F}_{u v}=\nabla_{u} A_{v}-\nabla_{v} A_{u}, \text { where } \tilde{\nabla}_{0}=\left(1+\hbar^{2} \beta g^{00} \nabla_{0}^{2}\right) \nabla_{0}, \tilde{\nabla}_{i}=\left(1-\hbar^{2} \beta g^{i i} \nabla_{i}^{2}\right) \nabla_{i},
\end{aligned}
$$

By using metric (49) into Eq. (52) and following the same procedure of Sec. IV, we obtained the modified tunneling rate for BTZ-like magnetic $\mathrm{BH}$ as

$$
\Gamma_{\mathcal{P}}=\exp \left[-2 \pi \frac{l^{2}\left(E-j \Omega_{h}-e A_{\varphi}\right)}{r_{+}^{2}-4 q_{m}^{2} \tau^{2} l^{4}}(1+\beta \chi)\right], \quad(\text { for } \hbar=1) .
$$

The modified tunneling probability of vector particles depends upon the energy $E$, charged potential of $\mathrm{BH} A_{\varphi}$, magnetic charge $q_{m}$, particle's charge $e$, curvature radius $\ell$, correction parameter $\beta$, angular momentum $j$ and angular velocity $\Omega_{h}$.

After utilizing Boltzmann factor $\Gamma_{\mathcal{P}}=\exp \left[\left(E-j \Omega_{h}-e A_{\varphi}\right) / T_{h}\right]$, the corresponding effective temperature for given $\mathrm{BH}$ can be calculated as follows

$$
T_{h}^{\prime}=\frac{r_{+}^{2}-4 q_{m}^{2} \tau^{2} l^{4}}{2 \pi l^{2} r_{+}(1+\beta \chi)}=T_{h}(1-\beta \chi)
$$

where

$$
T_{h}=\frac{r_{+}^{2}-4 q_{m}^{2} \tau^{2} l^{4}}{2 \pi l^{2} r_{+}}
$$

$T_{h}$ is the original Hawking temperature of BTZ-like magnetic BH. If we consider $\beta=0$, then the modified temperature $T_{h}^{\prime}$ convert into original Hawking temperature $T_{h}$ of BTZ-like magnetic BH [9]. The effective Hawking temperature depends upon magnetic charge $q_{m}$ of $\mathrm{BH}$, the curvature radius and correction parameter $\beta$. 


\section{QUANTUM CORRECTIONS OF SPIN-0 PARTICLES FOR QUINTESSENTIAL BH}

This section is devoted to study the quantum gravity effects on the tunneling of massive scalar particles from a $\mathrm{BH}$ with quintessential field.

We investigate the tunneling process of spin- 0 particles for given $\mathrm{BH}$ and calculate the modified tunneling probability and corresponding corrected Hawking temperature. For this purpose, we consider the modified Klein-Gordan equation with scalar field $\phi$ as [63]

$$
-(i \hbar)^{2} \partial_{t} \partial^{t} \phi=\left[m^{2}+(-i \hbar)^{2} \partial_{i} \partial^{i}\right]\left[1-2 \beta\left(m^{2}+(-i \hbar)^{2} \partial_{i} \partial^{i}\right)\right] \phi,
$$

The generalized Klein-Gordan equation in the background of quintessential BH metric (4), obtain the form

$$
\begin{aligned}
& \frac{\hbar^{2}}{X(r)} \frac{\partial^{2} \phi}{\partial t^{2}}-\frac{\hbar^{2}}{Z(r)} \frac{\partial^{2} \phi}{\partial x^{2}}-2 \hbar^{4} \beta Y(r) \frac{\partial^{2} \phi}{\partial r^{2}}\left[-Y(r) \frac{\partial^{2} \phi}{\partial r^{2}}\right]-2 \frac{\hbar^{4} \beta}{Z(r)} \frac{\partial^{2} \phi}{\partial x^{2}}\left[-\frac{1}{Z(r)} \frac{\partial^{2} \phi}{\partial x^{2}}\right] \\
& -\hbar^{2} Y(r) \frac{\partial^{2} \phi}{\partial r^{2}}+m^{2}\left(1-2 \beta m^{2}\right) \phi=0 .
\end{aligned}
$$

The wave function for spin-0 particles can be defined as follows

$$
\phi(t, r, x)=\left[\frac{i}{\hbar} I(t, r, x)\right]
$$

By putting Eq. (59) into Eq. (58) only for first order term in $\hbar$, we obtain

$$
\begin{aligned}
& \frac{1}{X(r)}\left(\frac{\partial I}{\partial t}\right)^{2}=Y(r)\left(\frac{\partial I}{\partial r}\right)^{2}+\frac{1}{Z(r)}\left(\frac{\partial I}{\partial x}\right)^{2}+m^{2}+\frac{\beta}{Z(r)^{2}}\left(\frac{\partial I}{\partial x}\right)^{4} \\
& -\beta\left[m^{4}-2 Y(r)^{2}\left(\frac{\partial I}{\partial r}\right)^{4}\right] .
\end{aligned}
$$

In order to solve this equation,we consider the following particle's action

$$
I(t, r, x)=-E t+j \varphi+R(r) .
$$

Here, $R(r)=R_{\circ}(r)+\beta R_{1}(r)$ [59], so the radial integral $R(r)$ gets the form

$$
R_{ \pm}(r)=\sqrt{\frac{E^{2}-X(r)\left(m^{2}+\frac{j^{2}}{Z(r)}\right)}{X(r)}}(1+\beta \Im) .
$$

After solving the above integral, we obtain the following relation

$$
R_{ \pm}(r)= \pm i \pi \frac{E}{X^{\prime}(r)}(1+\beta \Im)
$$

where $\Im>0$ can be given as

$$
\Im=\left(\frac{X(r)\left(m^{2}+\frac{j^{4}}{Z(r)^{2}}\right)}{E^{2}-X(r)\left(m^{2}+\frac{j^{2}}{Z(r)}\right)}-\frac{E^{2}-X(r)\left(m^{2}+\frac{j^{2}}{Z(r)}\right)}{X(r)}\right),
$$

Here, $R_{+}$and $R_{-}$are the radial functions for the outgoing/incoming particles, respectively. The tunneling probability for spin-0 particles at horizon can be given as follows

$$
\begin{aligned}
& \Gamma_{\mathcal{P}}=\frac{\mathcal{P}[\text { emission }]}{\mathcal{P}[\text { absorption }]} \simeq \exp \left[-\frac{4}{\hbar}\left(\operatorname{ImR} R_{+}\right)\right] \\
& \Gamma_{\mathcal{P}}=\exp \left[-\frac{4 \pi E}{\hbar X^{\prime}\left(r_{+}\right)}(1+\beta \Im)\right]=\exp \left[-\frac{8 \pi l^{2} E}{\hbar \rho^{2} r_{+}}(1+\beta \Im)\right]
\end{aligned}
$$


The corresponding corrected Hawking temperature for scalar particles can be calculated as follows

$$
T_{h}^{\prime}=\frac{\rho^{2} r_{+}}{8 \pi l^{2}(1+\beta \Im)}=T_{h}(1-\beta \Im),
$$

where

$$
T_{h}=\frac{\rho^{2} r_{+}}{8 \pi l^{2}}
$$

this is the original Hawking temperature of the given $\mathrm{BH}$. Equation (67) represents the effective Hawking temperature under quantum gravity effects.

\section{QUANTUM CORRECTIONS FOR BTZ-LIKE MAGNETIC BH}

In this section, we calculate the modified tunneling rate and corresponding effective Hawking temperature for BTZ-like BH in the presence of magnetic field. For this purpose, we follow the same procedure of previous Section VII and derive the modified tunneling probability for the given $\mathrm{BH}$ in the following form

$$
\Gamma_{\mathcal{P}}=\exp \left[-2 \pi \frac{l^{2} E}{r_{+}^{2}-4 q_{m}^{2} \tau^{2} l^{4}}(1+\beta \Im)\right] .
$$

The corresponding corrected Hawking temperature at $\mathrm{BH}$ horizon for spin-0 particles can be deduced in the form

$$
T_{h}^{\prime}=\frac{r_{+}^{2}-4 q_{m}^{2} \tau^{2} l^{4}}{2 \pi l^{2} r_{+}(1+\beta \Im)}=T_{h}(1-\beta \Im) .
$$

where $T_{h}=\frac{r_{+}^{2}-4 q_{m}^{2} \tau^{2} l^{4}}{2 \pi l^{2} r_{+}}$shows the standard Hawking temperature for BTZ-like magnetic BH. When we neglect quantum gravity effect i.e., $(\beta=0)$, then the corrected temperature reduced into standard Hawking temperature of BTZ-like BH.

\section{CONCLUSION}

In this paper, we have investigated the Hawking radiation process via quantum tunneling phenomenon for vector particles from $(2+1)$-dimensional $\mathrm{BH}$ with quintessence. In this regard, we have considered the Hamilton-Jacobi technique and WKB method to the field equation of massive vector particles. We have calculated the required tunneling rate of emitted particles and their corresponding Hawking temperature, $T_{h}$. Moreover, we have studied the quantum gravity effect on the tunneled spin-1 (vector) and spin-0 (scalar) particles from (2+1)-dimensional BHs in the background of quintessential and magnetic fields. Firstly, we consider the modified Proca and Klein-Gordon equations that describe the motion of spin- 1 and spin- 0 particles, respectively. Then, by utilizing the Hamilton-Jacobi method, the tunneling probabilities and effective Hawking temperatures $T_{h}^{\prime}$ for both type of particles from the given $\mathrm{BH}$ are calculated. We note that the Hawking temperature and corrected Hawking temperature not only based on the $\mathrm{BHs}$ properties but also depends on the radiated particles energy, mass, and angular momentum.

It is worth mentioning that when self-gravitational interactions are taken into account, corrected temperatures obtained in Eqs.(43) and (67) for quintessential BH and in Eqs.(55) \& (69) for BTZ-like magnetic BH looks identical for spin-1 and spin-0 particles. But the relation to angular momentum and mass are not entirely identical in these results. These temperatures also looked preserved over charge and energy. When we neglect the quantum gravity effects $(\beta=0)$, then the modified Hawking temperature of quintessential BH reduced into standard Hawking temperature of Eq. (24). Similarly, the corrected Hawking temperature of BTZ-like magnetic BH reduced into standard Hawking temperature of ref. [9].

It is important to mention here that the quantum corrections slow-down the increase in Hawking temperature during evaporation process. At the point when the mass of $\mathrm{BH}$ approaches to its minimum value, it stops radiating and $\mathrm{BH}$ remnant are left. 


\section{Acknowledgments}

This work was supported by Comisión Nacional de Ciencias y Tecnología of Chile through FONDECYT Grant $N^{\mathrm{o}}$ 3170035 (A. Ö.).

[1] S. W. Hawking, Commun. Math. Phys. 43, 199(1975); erratum-ibid, 46, 206(1976).

[2] S. W. Hawking, Nature, 248, 30(1974).

[3] S. W. Hawking, Phys. Rev. D 13, 191(1976).

[4] M. Sharif, W. Javed, Can. J. Phys. 90, 903(2012); ibid. Gen. Relativ. Gravit. 45, 1051(2013); ibid. J. Exp. Theor. Phys. 115, 782(2012); ibid. Proceedings of the 3rd Galileo, Xu Guangqi Meeting, Int. J. Mod. Phys.: Conference Series, 23, 271(2013);ibid. Proceedings of the 13th Marcel Grossmann Meeting (Stockholm, 2012), World Scientific, 3, 1950(2015); ibid. J. Korean. Phys. Soc. 57, 217(2010).

[5] M. Sharif, W. Javed, Eur. Phys. J. C 72, 1997(2012).

[6] M. Sharif, W. Javed, Can. J. Phys. 91, 43(2013).

[7] W. Javed, G. Abbas, R. Ali, Eur. Phys. J. C 77, 296(2017).

[8] W. Javed, R. Babar and A. Övgün, Mod. Phys. Lett. A 34, no. 09, 1950057 (2019).

[9] W. Javed, R. Ali, R. Babar and A. Övgün, Preprints 2019, 2019030194 (doi: 10.20944/preprints201903.0194.v3).

[10] W. Javed and R. Babar, Adv. High Energy Phys. 2019, 2759641(2019), https://doi.org/10.1155/2019/2759641.

[11] W. Javed, R. Ali, R. Babar and A. Övgün, Preprints 2019, 2019070172 (doi: 10.20944/preprints201907.0172.v1).

[12] V. E. Akhmedova, T. Pilling, A. de Gill and D. Singleton, Theor. Math. Phys. 163, 774 (2010).

[13] A. de Gill, D. Singleton, V. Akhmedova and T. Pilling, Am. J. Phys. 78, 685 (2010).

[14] T. Zhu, J. R. Ren and D. Singleton, Int. J. Mod. Phys. D 19, 159 (2010).

[15] M.Hossain Ali, Class.Quant.Grav. 24, 5849-5860 (2007).

[16] M.Hossain Ali, Int.J.Theor.Phys. 47, 2203-2217 (2008).

[17] M.Hossain Ali, Gen.Rel.Grav. 36, 1171-1181 (2004).

[18] E. T. Akhmedov, V. Akhmedova and D. Singleton, Phys. Lett. B 642, 124 (2006).

[19] V. Akhmedova, T. Pilling, A. de Gill and D. Singleton, Phys. Lett. B 666, 269 (2008).

[20] V. Akhmedova, T. Pilling, A. de Gill and D. Singleton, Phys. Lett. B 673, 227 (2009).

[21] X. M. Kuang, J. Saavedra and A. Övgün, Eur. Phys. J. C 77, no. 9, 613 (2017).

[22] K. Jusufi, I. Sakalli and A. Övgün, Gen. Rel. Grav. 50, no. 1, 10 (2018).

[23] A. Övgün, W. Javed and R. Ali, Adv. High Energy Phys. 2018, 3131620 (2018).

[24] P. A. Gonzalez, A. Övgün, J. Saavedra and Y. Vasquez, Gen. Rel. Grav. 50, no. 6, 62 (2018).

[25] I. Sakalli and A. Övgün, Eur. Phys. J. Plus 131, no. 6, 184 (2016).

[26] A. Övgün, Adv. High Energy Phys. 2017, 1573904 (2017).

[27] K. Jusufi and A. Övgün, Int. J. Theor. Phys. 56, no. 6, 1725 (2017).

[28] I. Sakalli and A. Ovgun, EPL 118, no. 6, 60006 (2017).

[29] K. Jusufi, A. Ovgun and G. Apostolovska, Adv. High Energy Phys. 2017, 8798657 (2017).

[30] T. M. He and J. Y. Zhang, Chin. Phys. Lett. 24, 3336 (2007).

[31] H. L. Li, S. Z. Yang, Q. Q. Jiang and D. J. Qi, Phys. Lett. B 641, 139 (2006).

[32] I. Sakalli and A. Ovgun, EPL 110, no. 1, 10008 (2015)

[33] I. Sakalli and A. Ovgun, J. Exp. Theor. Phys. 121, no. 3, 404 (2015)

[34] I. Sakalli and A. Ovgun, Eur. Phys. J. Plus 130, no. 6, 110 (2015)

[35] I. Sakalli and A. Ovgun, Astrophys. Space Sci. 359, no. 1, 32 (2015)

[36] I. Sakalli and A. Övgün, Gen. Rel. Grav. 48, no. 1, 1 (2016)

[37] G. R. Chen, S. Zhou and Y. C. Huang, Int. J. Mod. Phys. D 24, no. 01, 1550005 (2014).

[38] X. X. Zeng and S. Z. Yang, Chin. Phys. B 18, 462 (2009).

[39] R. Li and J. R. Ren, Phys. Lett. B 661, 370 (2008).

[40] H. L. Li and S. Z. Yang, EPL 79, no. 2, 20001 (2007).

[41] Q. Q. Jiang, S. Q. Wu and X. Cai, Phys. Lett. B 651, 58 (2007).

[42] E. T. Akhmedov, V. Akhmedova, T. Pilling and D. Singleton, Int. J. Mod. Phys. A 22, 1705 (2007).

[43] P. Kraus and F. Wilczek, Mod. Phys. Lett. A 09, 3713(1994).

[44] M. K. Parikh and F. Wilczek, Phys. Rev. Lett. 85, 5042(2000).

[45] P. Kraus and F. Wilczek, Nucl. Phys. B 433, 403(1995).

[46] P. Kraus and F. Wilczek, Nucl. Phys. B 437, 231(1995).

[47] M. K. Parikh, Phys. Lett. B 546, 189(2002).

[48] Angheben, M., Nadalini, M., Vanzo, L. and Zerbini, S.: J. High Energy Phys. 05(2005)014.

[49] A. Kempf, Journal of Mathematical Physics, 38, 1347(1997).

[50] A. F. Ali, S. Das, and E. C. Vagenas, Phys. Lett. B 678, 497(2009).

[51] S. Das, E.C. Vagenas, and A. F. Ali, Phys. Lett. B 690, 407(2010); Erratum to: Phys. Lett. B 692, 342(2010).

[52] B. Carr, J. Mureika, and P. Nicolini, Journal of High Energy Phys. 2015, 52(2015). 
[53] A. Kempf, G. Mangano and R.B. Mann, Phys. Rev. D 52, 1108(1995).

[54] J. de Oliveira and R. D. B. Fontana: Phys. Rev. D 98, 044005(2018).

[55] M. Banados, C. Teitelboim, and J. Zanelli, Phys. Rev. Lett. 69, 1849(1992).

[56] S. I. Kruglov, Mod. Phys. Lett. A 29, 1450203(2014).

[57] R. Kerner and R. B. Mann, Class. Quant. Grav. 25, 095014(2008).

[58] Li, X.Q.: Phys. Lett. B763(2016)80.

[59] Z.-Y. Liu and J.-R. Ren, Communications inTheoretical Physics, 62, 819(2014).

[60] Deyou Chen, Houwen Wu, Haitang Yang, Shuzheng Yang, Int. J. Mod. Phys. A 29 (2014) 1430054.

[61] Deyou Chen, Eur. Phys. J. C 74 (2014) 2687.

[62] G. Gecim and Y. Sucu, Mod. Phys. Lett. A 33, no. 28, 1850164 (2018).

[63] G. Gecim and Y. Sucu, Phys. Lett. B 773, 391 (2017).

[64] G. Gecim and Y. Sucu, Adv. High Energy Phys. 2018, 8728564 (2018).

[65] M.Hossain Ali and K. Sultana, Int.J.Theor.Phys. 56, no.7, 2279-2292 (2017).

[66] X. Q. Li, G.R. Chen, Phys. Lett. B 751, 34(2015).

[67] G. R. Chen, Y.C. Huang, Int. J. Mod. Phys. Rev. A 30, 1550083(2015).

[68] Z. Feng, Y. Chen, X. Zu, Astrophys. space Sci. 48, 359(2015).

[69] T. I. Singh, I. A. Meitei, K. Y. Singh, Astrophys. Space Sci. 361, 103(2015).

[70] T. Shivalingaswamy, B.A. Kagali, Eur. J. Phys. Education. 2, 1309(2011).

[71] S. Kanzi and I. Sakalli, arXiv:1905.00477 [hep-th].

[72] X.Q. Li, G.R. Chen, Phys. Lett. B751, 34(2015).

[73] G. Gecim and Y. Sucu, Gen. Rel. Grav. 50, no. 12, 152 (2018)

[74] X. He and W. Liu, Phys. Lett. B 653, 330 (2007).

[75] A. Övgün, Int. J. Theor. Phys. 55, no. 6, 2919 (2016)

[76] A. Övgün and K. Jusufi, Eur. Phys. J. Plus 131, no. 5, 177 (2016).

[77] S. K. Modak, Phys. Lett. B 671, 167 (2009).

[78] I. A. Meitei, T. I. Singh, S. G. Devi, N. P. Devi and K. Y. Singh, Int. J. Mod. Phys. A 33, no. 12, 1850070 (2018).

[79] K. Jusufi and A. Övgün, Astrophys. Space Sci. 361, no. 7, 207 (2016).

[80] I. Sakalli and A. Övgün, J. Astrophys. Astron. 37, 21 (2016).

[81] I. Sakalli, A. Övgün and K. Jusufi, Astrophys. Space Sci. 361, no. 10, 330 (2016).

[82] A. Övgün and K. Jusufi, Eur. Phys. J. Plus 132, no. 7, 298 (2017).

[83] X. M. Kuang, B. Liu and A. Övgün, Eur. Phys. J. C 78, no. 10, 840 (2018).

[84] M. Rizwan, M. Z. Ali and A. Övgün, doi:10.1142/S0217732319501840, arXiv:1812.01983 [physics.gen-ph].

[85] A. G. Riess, et al., Astron. J. 116, 1009(1998).

[86] J. H. Chen and Y. J. Wang, Commun. Theor. Phys. 50, 101(2008).

[87] S. B. Chen, B. Wang, and R. K. Su, Phys. Rev. D 77, 124011(2008).

[88] Y. J. Wang and Z. M. Tang, Astrophys. and S. S. 282, 237(2002).

[89] M. Banados, C. Teitelboim and J. Zanelli, Phys. Rev. Lett. 69, 1849(1992).

[90] S. S. Gubser, I. R. Klebanov and A. W. Peet, Phys. Rev. D 54, 3915(1996). 